\section{BIOMARKERS IN CUTANEOUS MELANOMA: CASTLES IN THE SKY AND USEFUL DEVELOPMENTS}

\author{
Peter Ferguson ${ }^{1,2}$ \\ ${ }^{1}$ Department of Tissue Pathology and Diagnostic Oncology, \\ Royal Prince Alfred Hospital, New South Wales Health \\ Pathology, Sydney, NSW, Australia; and ${ }^{2}$ Melanoma Institute \\ Australia, The University of Sydney, North Sydney, NSW, \\ Australia
}

Recent advances in systemic therapy have improved outcomes for patients with advanced stage cutaneous melanoma. This has led to an increased clinical need for biomarkers to identify patients with early stage disease who are likely to benefit from adjuvant treatment. Prognostic calculators can refine prediction of the risk of recurrence by incorporating additional clinicopathological parameters that are not included in melanoma staging by the 8th edition of the AJCC Cancer Staging Manual. Novel prognostic biomarkers are being developed through a range of molecular methods, including gene expression profiling, targeted sequencing and proteomics. There is also a need for improved predictive biomarkers, to identify patients whose melanomas will respond to immunotherapy and distinguish those who are at risk of toxicity without therapeutic benefit. This presentation provides a snapshot of recent developments and future challenges in the development and application of biomarkers in the management of primary cutaneous melanoma.

\section{PREDICTING ENDOCRINE SENSITIVITY IN BREAST CANCER}

Cameron E. Snell ${ }^{1,2}$, Deborah Smith ${ }^{1,2}$, Madeline Gough ${ }^{1,2}$, Cheng Liu $^{2}$, Kathryn Middleton ${ }^{3,5}$, Christopher Pyke ${ }^{4,5}$, Catherine Shannon ${ }^{3,5}$, Natasha Woodward ${ }^{3,5}$

${ }^{1}$ Cancer Pathology Research Group, Mater Research Institute - The University of Queensland, Translational Research Institute, Qld, Australia; ${ }^{2}$ Department of Anatomical Pathology, Mater Pathology, Mater Hospital Brisbane, Qld, Australia; ${ }^{3}$ Department of Medical Oncology, Mater Hospital Brisbane, Qld, Australia; ${ }^{4}$ Department of Breast and Endocrine Surgery, Mater Hospital Brisbane, Qld, Australia; and ${ }^{5}$ Mater Research Institute - The University of Queensland, Translational Research Institute, Qld, Australia

Approximately $75 \%$ of breast cancers express oestrogen receptors (ER), progesterone receptors (PR) or both. Endocrine therapy is a cornerstone of systemic therapy for ER+ breast cancer in both the adjuvant and metastatic setting. Despite adjuvant endocrine therapy, over $20 \%$ of women with earlystage disease suffer a relapse which can occur years or decades after diagnosis. ER expression only poorly predicts outcome and better predictive markers of response or resistance are required. Molecular signature assays such as Oncotype Dx, Prosigna, Mammaprint and Endopredict have produced information on prognosis and aid in the decision as to whether patients should have adjuvant chemotherapy in addition to surgery and endocrine therapy.

We have recently shown that a physical interaction between ER and PR exists and is potentiated by the presence of oestrogen and progesterone. ${ }^{1} \mathrm{PR}$ alters the interaction between ER and chromatin to change the transcriptional output of the ER complex. We have quantified interactions between ER and PR in breast cancer to demonstrate that low ER/PR interactions are associated with relapse on adjuvant aromatase inhibitors. We are currently utilising a novel patient-derived explant (PDE) culture model to measure the sensitivity of tumours to oestrogen, progesterone, tamoxifen and targeted treatments including CDK4/6 inhibitors. Reference

1. Snell CE, Gough M, Liu C, et al. Improved relapse-free survival on aromatase inhibitors in breast cancer is associated with interaction between oestrogen receptor-alpha andprogesterone receptor-b. Br J Cancer 2018; 119: 1316-25.

\section{BACKGROUND, TESTING METHODS, AND LABORATORY APPROACHES TO SARS CORONAVIRUS-2 (SARS-COV-2) AND COVID19}

William D. Rawlinson ${ }^{1,2}$, Sebastiaan van $\mathrm{Hal}^{3}$, Malinna Yeang ${ }^{1}$, Anna Condylios ${ }^{1}$, Zin Naing ${ }^{1}$, Jane-Phan $\mathrm{Au}^{1,2}$, Mariana Ruiz da Silva ${ }^{1,2}$, Charles Foster ${ }^{1,2}$

${ }^{1}$ Serology and Virology Division, NSW Health Pathology Microbiology, SEALS Prince of Wales Hospital, NSW, Australia; ${ }^{2}$ Schools of Women's and Children's Health, Medicine and Biotechnology and Biomolecular Sciences, University of New South Wales, Sydney, NSW, Australia; and ${ }^{3}$ Department of Microbiology and Infectious Diseases, NSW Health Pathology, Royal Prince Alfred Hospital, NSW, Australia

Introduction: A pandemic is an epidemic occurring worldwide, crossing international boundaries and affecting many people. The spread of SARS-CoV-2 from Hubei province in China globally has led to rapid changes in our approaches to diagnosis, with rapid use of molecular methods such as whole genome sequencing (WGS) to characterise mutations, and deployment of novel therapeutics including mRNA vaccines and monoclonal antibodies.

Methods: New methods for diagnosis, including WGS, and different approaches to emerging mutations in virus variants of concern (VOC) will be discussed.

Results and conclusions: Diagnosis now uses standard molecular methods including commercial and inhouse techniques, more recently rapid antigen detection, and research into rapid smartphone based nanoparticle assays. Laboratory practices of sample pooling, reflex testing using WGS, and workflow changes are now routine.

The use of rapid WGS techniques, ${ }^{1}$ and available Australian data, ${ }^{2,3}$ have informed assessment of the accuracy and utility of these methods in the diagnostic laboratory. Assessment of changes in PCR primer binding sites, and of the influence of how sequence 